\title{
On decoupling inequalities and percolation of excursion sets of the Gaussian free field
}

\author{
Serguei Popov ${ }^{1} \quad$ Balázs Ráth ${ }^{2}$
}

February 5, 2015

\begin{abstract}
${ }^{1}$ Department of Statistics, Institute of Mathematics, Statistics and Scientific Computation, University of Campinas - UNICAMP, rua Sérgio Buarque de Holanda 651, 13083-859, Campinas SP, Brazil

e-mail: popov@ime.unicamp.br

${ }^{2}$ Budapest University of Technology, Institute of Mathematics, MTA-BME Stochastics Research Group, 1 Egry József u., 1111 Budapest, Hungary.

e-mail: rathb@math.bme.hu
\end{abstract}

\begin{abstract}
We prove decoupling inequalities for the Gaussian free field on $\mathbb{Z}^{d}$, $d \geq 3$. As an application, we obtain exponential decay (with logarithmic correction for $d=3$ ) of the connectivity function of excursion sets for large values of the threshold.
\end{abstract}

Keywords: percolation, Gaussian free field, simple random walk, random interlacements

AMS 2000 subject classifications: Primary 60K35, 82B43.

\section{Correlation and decoupling inequalities}

Let us denote by $\|x\|$ the Euclidean norm of $x \in \mathbb{Z}^{d}$. The Gaussian free field $(\mathrm{GFF})$ on $\mathbb{Z}^{d}, d \geq 3$, is a centered Gaussian field $\varphi=\left(\varphi_{x}\right)_{x \in \mathbb{Z}^{d}} \in \mathbb{R}^{\mathbb{Z}^{d}}$ under the probability measure $\mathbb{P}$ with covariance $\mathbb{E}\left(\varphi_{x} \varphi_{y}\right)=g(x, y)$ for all $x, y \in \mathbb{Z}^{d}$, where $g(\cdot, \cdot)$ denotes the Green function of the simple random walk on $\mathbb{Z}^{d}$. The random field $\varphi$ exhibits long-range correlations, since

$$
c_{g}\|x-y\|^{2-d} \leq g(x, y) \leq C_{g}\|x-y\|^{2-d}, \quad x \neq y
$$

for some $0<c_{g}(d) \leq C_{g}(d)<\infty$, see [6, Theorem 1.5.4]. 
The goal of this section is to quantify the dependence between configurations of the GFF, supported on disjoint (and, usually, distant) sets. Decoupling inequalities of this kind are useful tools in the study of percolation of the excursion sets of the GFF (see [10, Proposition 2.2] and [2, Lemma 2.6]) and the vacant set of random interlacements (see [13, Theorem 2.6]). When starting to work on the subject of the present paper, our initial aim was to find the GFF counterpart of the decoupling inequalities proved for random interlacements in [8, Theorem 1.1] (see Remark 1.5 for further discussion), but our Theorem 1.2 actually gives a stronger, conditional form of decoupling inequalities for events defined in terms of the GFF. In Section 2 we give an application of the decoupling inequalities stated in this section.

If $K, K^{\prime} \subseteq \mathbb{Z}^{d}$, we define

$$
\operatorname{dist}\left(K, K^{\prime}\right)=\min _{x \in K, y \in K^{\prime}}\|x-y\|, \quad \operatorname{diam}(K)=\sup _{x, y \in K}\|x-y\| .
$$

With a slight abuse of notation, for $K \subset \mathbb{Z}^{d}$ we write $\varphi_{K}:=\left(\varphi_{x}\right)_{x \in K}$ for the GFF restricted to $K$. We say that a (measurable) function $f: \mathbb{R}^{\mathbb{Z}^{d}} \rightarrow \mathbb{R}$ is supported on $K$ if for any $\eta, \eta^{\prime} \in \mathbb{R}^{\mathbb{Z}^{d}}$ such that $\eta_{K}=\eta_{K}^{\prime}$ it holds that $f(\eta)=f\left(\eta^{\prime}\right)$. Let us also define

$$
\operatorname{Cov}\left(f_{1}(\varphi), f_{2}(\varphi)\right)=\mathbb{E}\left(f_{1}(\varphi) f_{2}(\varphi)\right)-\mathbb{E} f_{1}(\varphi) \mathbb{E} f_{2}(\varphi) .
$$

If $K$ is a finite subset of $\mathbb{Z}^{d}$, we define the capacity of $K$ by the formula

$$
\operatorname{cap}(K)=\sum_{x \in K} P_{x}\left[\widetilde{H}_{K}=+\infty\right]
$$

where $P_{x}$ is the law of simple random walk $X$ on $\mathbb{Z}^{d}$ started from $x \in \mathbb{Z}^{d}$ and $\widetilde{H}_{K}=\min \left\{n \geq 1: X_{n} \in K\right\}$ is the hitting time of the set $K$.

First, we formulate the following result about correlations of functions supported on disjoint finite sets, which is in the spirit of the basic correlation inequality for random interlacements, see [12, (2.15)].

Proposition 1.1. Let $d \geq 3$. There exist constants $0<c_{d} \leq C_{d}<+\infty$ that depend only on $d$ such that if $K_{1}, K_{2}$ are disjoint finite subsets of $\mathbb{Z}^{d}$ and

$$
\operatorname{dist}\left(K_{1}, K_{2}\right) \geq \max \left\{\operatorname{diam}\left(K_{1}\right), \operatorname{diam}\left(K_{2}\right)\right\},
$$

then

$c_{d} \frac{\left(\operatorname{cap}\left(K_{1}\right) \operatorname{cap}\left(K_{2}\right)\right)^{1 / 2}}{\operatorname{dist}\left(K_{1}, K_{2}\right)^{d-2}} \leq \sup _{f_{1}, f_{2}} \operatorname{Cov}\left(f_{1}(\varphi), f_{2}(\varphi)\right) \leq C_{d} \frac{\left(\operatorname{cap}\left(K_{1}\right) \operatorname{cap}\left(K_{2}\right)\right)^{1 / 2}}{\operatorname{dist}\left(K_{1}, K_{2}\right)^{d-2}}$,

where the supremum is taken over $[0,1]$-valued functions $f_{1}, f_{2}: \mathbb{R}^{\mathbb{Z}^{d}} \rightarrow[0,1]$, and where $f_{1}$ is supported on $K_{1}$ and $f_{2}$ is supported on $K_{2}$. 
Let us remark that the assumption (1.2) is only used in the proof the lower bound of (1.3).

While the above result does indeed give the right order of decay of correlations, it is not always the right tool one looks for. The reason is that the covariance decreases polynomially in distance, which makes renormalization arguments more difficult. One can circumvent this problem by using the method of sprinkling, which has been effectively applied to produce powerful decoupling inequalities for the excursion sets of the GFF (see [10, Proposition 2.2] and [2, Lemma 2.6]) and the vacant set of random interlacements (see [13, Section 2]).

To explain this approach, we need more definitions. Write $\eta \leq \eta^{\prime}$ if $\eta_{x} \leq \eta_{x}^{\prime}$ for all $x \in \mathbb{Z}^{d}$. A function $f: \mathbb{R}^{\mathbb{Z}^{d}} \rightarrow \mathbb{R}$ is called increasing if $\eta \leq \eta^{\prime}$ implies $f(\eta) \leq f\left(\eta^{\prime}\right)$, and decreasing if $(-f)$ is increasing. For $\eta \in \mathbb{R}^{\mathbb{Z}^{d}}$ and $a \in \mathbb{R}$ we use the shorthand $\eta+a$ for the configuration defined by $(\eta+a)_{x}=\eta_{x}+a, x \in \mathbb{Z}^{d}$.

Let us fix two disjoint sets $K_{1}, K_{2} \subset \mathbb{Z}^{d}$, such that $K_{1}$ is finite. It is known (see [14, Proposition 2.3] and [10, Lemma 1.2]) that $\mathbb{P}$-a.s. there exists a decomposition

$$
\varphi=\tilde{\varphi}+h
$$

into a sum of independent Gaussian fields, where $\tilde{\varphi}$ is a centered field such that $\tilde{\varphi}_{K_{1}} \equiv 0$, and

$$
h_{x}=\sum_{y \in K_{1}} P_{x}\left[H_{K_{1}}<\infty, X_{H_{K_{1}}}=y\right] \cdot \varphi_{y}, \quad \mathbb{P} \text {-a.s. }
$$

where $H_{K_{1}}=\min \left\{n \geq 0: X_{n} \in K_{1}\right\}$ is the entrance time of the random walk to the set $K_{1}$. Note that $h$ is measurable with respect to the sigma-algebra generated by $\varphi_{K_{1}}$ and that one has $h_{K_{1}} \equiv \varphi_{K_{1}}$.

For $\delta>0$, define the $\sigma\left(\varphi_{K_{1}}\right)$-measurable event

$$
G_{\delta}=\left\{\sup _{x \in K_{2}}\left|h_{x}\right| \leq \frac{\delta}{2}\right\}
$$

Our main result is about the conditional decoupling inequalities:

Theorem 1.2. Assume that $f_{2}: \mathbb{R}^{\mathbb{Z}^{d}} \rightarrow[0,1]$ is increasing and supported on $K_{2}$. For all $\delta>0$ it $\mathbb{P}$-a.s. holds that

$$
\left(\mathbb{E}\left(f_{2}(\varphi-\delta)\right)-\mathbb{P}\left[G_{\delta}^{c}\right]\right) \mathbb{1}_{G_{\delta}} \leq \mathbb{E}\left(f_{2}(\varphi) \mid \varphi_{K_{1}}\right) \mathbb{1}_{G_{\delta}} \leq\left(\mathbb{E}\left(f_{2}(\varphi+\delta)\right)+\mathbb{P}\left[G_{\delta}^{c}\right]\right) \mathbb{1}_{G_{\delta}}
$$

An (almost) immediate consequence of this result is the following 
Corollary 1.3. Assume that $f_{2}: \mathbb{R}^{\mathbb{Z}^{d}} \rightarrow[0,1]$ is increasing and supported on $K_{2}$, and $f_{1}: \mathbb{R}^{\mathbb{Z}^{d}} \rightarrow[0,1]$ is any function supported on $K_{1}$. Then for all $\delta>0$ we have

$$
\mathbb{E} f_{1}(\varphi) \mathbb{E} f_{2}(\varphi-\delta)-2 \mathbb{P}\left[G_{\delta}^{c}\right] \leq \mathbb{E} f_{1}(\varphi) f_{2}(\varphi) \leq \mathbb{E} f_{1}(\varphi) \mathbb{E} f_{2}(\varphi+\delta)+2 \mathbb{P}\left[G_{\delta}^{c}\right]
$$

It is straightforward to see that the corresponding results (with obvious changes) also hold if $f_{2}$ is a decreasing function.

We refer to the quantity $\delta$ in (1.7)-(1.8) as the amount of sprinkling. In Proposition 1.4 below we shall see that the term $\mathbb{P}\left[G_{\delta}^{c}\right]$ decreases quite fast as dist $\left(K_{1}, K_{2}\right)$ increases; so one can decrease the correlation term of Proposition 1.1 at the cost of "changing the level" of the field for the monotone function $f_{2}$.

The next proposition tells us how to choose the amount of sprinkling $\delta$ if we want a useful upper bound for $\mathbb{P}\left[G_{\delta}^{c}\right]$. Let us denote by $|K|$ the cardinality of $K \subset \mathbb{Z}^{d}$. For any $K \subseteq \mathbb{Z}^{d}$ we define

$$
\begin{aligned}
& K^{(\geq s)}=\left\{y \in \mathbb{Z}^{d}: \operatorname{dist}(y, K) \geq s\right\} \\
& K^{(=s)}=\left\{y \in \mathbb{Z}^{d}: s \leq \operatorname{dist}(y, K)<s+1\right\} .
\end{aligned}
$$

Note that a nearest-neighbor walk from $K$ to $K^{(\geq s)}$ must pass through $K^{(=s)}$.

Having fixed the disjoint subsets $K_{1}$ and $K_{2}$ of $\mathbb{Z}^{d}$, let us define $s=$ $\operatorname{dist}\left(K_{1}, K_{2}\right)>0$ and the auxiliary sets $H_{1}, H_{2} \subseteq \mathbb{Z}^{d}$ in the following way:

- if $\operatorname{diam}\left(K_{1}\right) \leq \operatorname{diam}\left(K_{2}\right)$, define $H_{2}=K_{1}^{(\geq s)}$ and $H_{1}=H_{2}^{(\geq s)}$,

- if $\operatorname{diam}\left(K_{1}\right)>\operatorname{diam}\left(K_{2}\right)$, define $H_{1}=K_{2}^{(\geq s)}$ and $H_{2}=H_{1}^{(\geq s)}$.

With these definitions we have

$$
K_{i} \subseteq H_{i}, \quad H_{3-i}=H_{i}^{(\geq s)}, \quad\left|H_{i}^{(=s)}\right|<\infty, \quad i \in\{1,2\} .
$$

Let us define

$$
g_{s}=\sup _{y:\|y\| \geq s} g(0, y)
$$

Proposition 1.4. Denote by $s=\operatorname{dist}\left(K_{1}, K_{2}\right)>0$. Then

$$
\mathbb{P}\left[G_{\delta}^{c}\right] \leq 2\left|H_{1}^{(=s)}\right| \exp \left(-\frac{\delta^{2}}{8 g_{s}}\right)
$$


Remark 1.5. Let us compare the inequality obtained from the combination of (1.8) and (1.10) with the main decoupling result (Theorem 2.1) of [8]. Observing that $g_{s}=\mathcal{O}\left(s^{2-d}\right)$ by (1.1), one can note the similarity of the expression in the exponent in the right-hand side of (1.10) with that in the error term in [8, Theorem 2.1]. Also, let $r$ be the minimum of the Euclidean diameters of $K_{1}$ and $K_{2}$. Then, one can (very crudely) bound $\left|H_{1}^{(=s)}\right|$ by const $\times(r+s)^{d}$ and again observe the similarity with the error term in [8, Theorem 2.1].

Before we prove the results stated above in Section 3, we give an application of our decoupling inequality in Section 2 .

\section{Connectivity decay for percolation of ex- cursion sets}

For any $h \in \mathbb{R}$, we define the excursion set above level $h$ as

$$
E_{\varphi}^{\geq h}=\left\{x \in \mathbb{Z}^{d}: \varphi_{x} \geq h\right\} .
$$

We view $E_{\bar{\varphi}}^{\geq h}$ as a random subgraph of $\mathbb{Z}^{d}$, and, naturally, one may be interested in studying its percolation properties. Let us write $\{x \stackrel{\geq h}{\longleftrightarrow} y\}$ for the event when $x, y \in \mathbb{Z}^{d}$ are connected in $E_{\bar{\varphi}}^{\geq h}$. As an application of Corollary 1.3, in this section we establish a result on the decay of the connectivity function of the excursion set $E_{\bar{\varphi}}^{\geq h}$.

In [1] (for $d=3$ ) and in [10] (for all $d \geq 3$ ) it was shown that there exists $h_{*}=h_{*}(d) \in[0,+\infty)$ such that $E_{\bar{\varphi}}^{\geq h}$ percolates for $h<h_{*}$ and does not percolate for $h>h_{*}$; moreover, it was also proved that $h_{*}(d)>0$ for all sufficiently large $d$. Further developments regarding percolation of $E_{\bar{\varphi}}^{\geq h}$ and its connection to interlacement percolation (based on the isomorphism theorem [15]) can be found in [7, 11].

In [10, (0.6)] the authors define another critical parameter $h_{* *} \geq h_{*}$ as the threshold above which one has at least polynomial decay of the probabilities of certain crossing events:

$$
h_{* *}(d)=\inf \left\{h \in \mathbb{R} ; \text { for some } \alpha>0, \lim _{L \rightarrow \infty} L^{\alpha} \mathbb{P}[B(0, L) \stackrel{\geq h}{\longleftrightarrow} S(0,2 L)]=0\right\},
$$

where the event $\{B(0, L) \stackrel{\geq h}{\longleftrightarrow} S(0,2 L)\}$ refers to the existence of a nearestneighbour path in $E_{\bar{\varphi}}^{\geq h}$ connecting $B(0, L)$, the ball of radius $L$ around 0 in the $\ell^{\infty}$-norm, to $S(0,2 L)$, the $\ell^{\infty}$-sphere of radius $2 L$ around 0 . 
In [10, Theorem 2.6] they prove that $h_{* *}(d)<\infty$ for all $d \geq 3$, and also give a stretched exponential upper bound for the connectivity function $\mathbb{P}[0 \stackrel{\geq h}{\longleftrightarrow} x]$ as $\|x\| \rightarrow \infty$ for $h>h_{* *}$. In the next theorem, we further weaken the definition of $h_{* *}$ and, more importantly, we improve on the stretched exponential bound for values of $h$ above $h_{* *}$.

Theorem 2.1. For $d \geq 4$, given $h>h_{* *}(d)$, there exist positive constants $\gamma_{1}=\gamma_{1}(d, h)$ and $\gamma_{2}=\gamma_{2}(d, h)$ such that

$$
\mathbb{P}[0 \stackrel{\gtrless h}{\longleftrightarrow} x] \leq \gamma_{1} \exp \left\{-\gamma_{2}\|x\|\right\}, \text { for every } x \in \mathbb{Z}^{d} .
$$

If $d=3$ and $h>h_{* *}(3)$, then for any $b>1$ there exist $\gamma_{1}^{\prime}=\gamma_{1}^{\prime}(h, b)$ and $\gamma_{2}^{\prime}=\gamma_{2}^{\prime}(h, b)$ such that

$$
\mathbb{P}[0 \stackrel{\geq h}{\longleftrightarrow} x] \leq \gamma_{1}^{\prime} \exp \left\{-\gamma_{2}^{\prime} \frac{\|x\|}{\log ^{3 b}\|x\|}\right\}, \text { for every } x \in \mathbb{Z}^{d} .
$$

Moreover, we show that the quantity $h_{* *}$ can be defined as

$$
h_{* *}=\inf \left\{h>0 ; \liminf _{L \rightarrow \infty} \mathbb{P}\left[[0, L]^{d} \stackrel{\geq h}{\longleftrightarrow} \partial[-L, 2 L]^{d}\right]<\frac{7}{2 d \cdot 21^{d}}\right\} .
$$

Proof of Theorem 2.1. The reader may have noticed that the above result is a copy of the statement of [8, Theorem 3.1], with obvious notational changes. Indeed, as observed in [8, Remark 3.4], the proof of that theorem can be adapted to any percolation model which satisfies certain monotonicity and decoupling properties, that the excursion sets of the GFF do possess.

More specifically, let us denote by $\mathbb{P}_{h}$ the law of the excursion set $E_{\bar{\varphi}}^{\geq h}$. Then (1.8) and (1.10) imply that for any increasing events $A_{1}, A_{2}$ that depend on disjoint boxes of size $r$ within distance at least $s$ from each other, we have

$$
\mathbb{P}_{h}\left[A_{1} \cap A_{2}\right] \leq \mathbb{P}_{h-\delta}\left[A_{1}\right] \mathbb{P}_{h-\delta}\left[A_{2}\right]+C(r+s)^{d} \exp \left(-C^{\prime} \delta^{2} s^{d-2}\right)
$$

This decoupling inequality is a special case of the one in [8, Remark 3.4], the proof of this result is also practically a copy of the proof of [8, Theorem 3.1].

It is important to observe that (2.4) may be seen as a partial replacement of the BK inequality, which is very useful for proving exponential decay of crossing probabilities in the subcritical phase of classical (Bernoulli) percolation, see e.g. Section 5.2 of [4]. While the "pure" BK inequality generally does not hold in the dependent percolation models we mentioned here, inequalities similar to (2.4) are still very useful, even though they usually involve 
sprinkling and the additive "error" term. It turns out that, for renormalization arguments, this additive term is pivotal, in the sense that the smaller it is, the better results one obtains for the decay of the probabilities of crossing events. In fact one can even achieve exponential decay (if $d \geq 4$ ) in polynomially correlated percolation models without the BK inequality.

Another important observation is that the question whether $h_{*}=h_{* *}$ is still open (as well as the corresponding question for random interlacements). In our opinion, the conditional decoupling result of our Theorem 1.2 might help in proving it; in fact, it did help in the proof of the fact that $h_{*}(d) / h_{* *}(d) \rightarrow 1$ as $d \rightarrow \infty$, see [3].

\section{Proofs of the decoupling results}

We start by deducing the correlation bounds with no sprinkling from the corresponding general results of [5, Chapter 10].

Proof of Proposition 1.1. First note that a function $f: \mathbb{R}^{\mathbb{Z}^{d}} \rightarrow \mathbb{R}$ is supported on $K$ if and only if $f$ is $\sigma\left(\varphi_{K}\right)$-measurable.

Denote by $\mathbf{G}$ the Gaussian Hilbert space that arises as the closure of vector space of linear combinations of $\varphi_{x}, x \in \mathbb{Z}^{d}$ under the norm $\|X\|=$ $\sqrt{\langle X, X\rangle}$ given by the inner product $\langle X, Y\rangle=\operatorname{Cov}(X, Y)$.

Denote by $\mathbf{H}$ and $\mathbf{K}$ the subspaces of $\mathbf{G}$ spanned by linear combinations of $\varphi_{x}, x \in K_{1}$ and $\varphi_{x}, x \in K_{2}$, respectively. Let us introduce the sigmaalgebras $\mathcal{F}=\sigma(\mathbf{H})=\sigma\left(\varphi_{K_{1}}\right)$ and $\mathcal{G}=\sigma(\mathbf{K})=\sigma\left(\varphi_{K_{2}}\right)$.

Recall from [5, Definitions 10.5, 10.6] the notion of the strong mixing coefficient $\alpha(\cdot, \cdot)$ and the maximal correlation coefficient $\rho(\cdot, \cdot)$ :

$$
\begin{aligned}
\alpha(\mathbf{H}, \mathbf{K}) & =\alpha(\mathcal{F}, \mathcal{G})=\sup _{A \in \mathcal{F}, B \in \mathcal{G}}|\mathbb{P}[A \cap B]-\mathbb{P}[A] \mathbb{P}[B]|, \\
\rho(\mathbf{H}, \mathbf{K}) & =\rho(\mathcal{F}, \mathcal{G})=\sup _{X \in L^{2}(\mathcal{F}), Y \in L^{2}(\mathcal{G})} \frac{\operatorname{Cov}(X, Y)}{\sqrt{\operatorname{Var}(X) \operatorname{Var}(Y)}} .
\end{aligned}
$$

With this notation we have

$$
\alpha(\mathbf{H}, \mathbf{K}) \stackrel{(*)}{\leq} \sup _{f_{1}, f_{2}} \operatorname{Cov}\left(f_{1}(\varphi), f_{2}(\varphi)\right) \stackrel{(* *)}{\leq} \rho(\mathbf{H}, \mathbf{K})
$$

where the supremum is taken over $[0,1]$-valued functions $f_{1}, f_{2}: \mathbb{R}^{\mathbb{Z}^{d}} \rightarrow[0,1]$, and where $f_{1}$ is supported on $K_{1}$ and $f_{2}$ is supported on $K_{2}$. Indeed, $(*)$ follows if we choose $f_{1}=\mathbb{1}_{A}$ and $f_{2}=\mathbb{1}_{B}$ (or $f_{2}=\mathbb{1}_{B^{c}}$ if $\operatorname{Cov}\left(\mathbb{1}_{A}, \mathbb{1}_{B}\right)<0$ ) and $(* *)$ follows because $\operatorname{Var}\left(f_{1}\right), \operatorname{Var}\left(f_{2}\right) \leq 1$. 
Denote by $P_{\mathbf{K}}: \mathbf{G} \rightarrow \mathbf{K}$ the orthogonal projection to the subspace $\mathbf{K}$ and by $P_{\mathbf{H K}}: \mathbf{H} \rightarrow \mathbf{K}$ the restriction of $P_{\mathbf{K}}$ to $\mathbf{H}$. Similarly, let $P_{\mathbf{H}}$ denote the projection to $\mathbf{H}$ and $P_{\mathbf{K H}}$ its restriction to $\mathbf{K}$. Denote by $\|\cdot\|$ the operator norm of linear operators on (subspaces of) $\mathbf{G}$.

[5, Theorem 10.11] states that $\rho(\mathbf{H}, \mathbf{K})=\left\|P_{\mathbf{H K}}\right\|$ and [5, Remark 10.1(ii), Theorem 10.13] imply that $\frac{1}{2 \pi}\left\|P_{\mathbf{H K}}\right\| \leq \alpha(\mathbf{H}, \mathbf{K})$. Combining these results with (3.1) we see that in order to prove Proposition 1.1 we only need to show that there exist constants $0<c_{d}^{\prime} \leq C_{d}^{\prime}<+\infty$ that depend only on $d$ such that if $K_{1}, K_{2}$ satisfy (1.2) then we have

$$
c_{d}^{\prime} \frac{\left(\operatorname{cap}\left(K_{1}\right) \operatorname{cap}\left(K_{2}\right)\right)^{1 / 2}}{\operatorname{dist}\left(K_{1}, K_{2}\right)^{d-2}} \leq\left\|P_{\mathbf{H K}}\right\| \leq C_{d}^{\prime} \frac{\left(\operatorname{cap}\left(K_{1}\right) \operatorname{cap}\left(K_{2}\right)\right)^{1 / 2}}{\operatorname{dist}\left(K_{1}, K_{2}\right)^{d-2}} .
$$

First note that the adjoint of $P_{\mathbf{H K}}$ is $P_{\mathbf{K H}}$ (see [5, Remark 10.1]), thus if we define $A: \mathbf{H} \rightarrow \mathbf{H}$ by $A=P_{\mathbf{K H}} P_{\mathbf{H K}}$, then $A$ is self-adjoint and we have (see, for example, [5, Appendix $\mathrm{H}]$ )

$$
\left\|P_{\mathbf{K H}}\right\|=\left\|P_{\mathbf{H K}}\right\|=\sqrt{\|A\|} .
$$

Note that we have

$$
\left\|P_{\mathbf{H K}}\right\|=\sup _{X \in \mathbf{H}, Y \in \mathbf{K}} \frac{\left\langle P_{\mathbf{K}}(X), Y\right\rangle}{\|X\|\|Y\|}=\sup _{X \in \mathbf{H}, Y \in \mathbf{K}} \frac{\langle X, Y\rangle}{\|X\|\|Y\|} .
$$

Now $X \in \mathbf{H}$ if and only if $X=\sum_{x \in K_{1}} \alpha_{x} \varphi_{x}$ for some $\alpha \in \mathbb{R}^{K_{1}}$ and $Y \in \mathbf{K}$ if and only if $Y=\sum_{y \in K_{2}} \beta_{y} \varphi_{y}$ for some $\beta \in \mathbb{R}^{K_{2}}$, thus we can use this coordinatization and $\operatorname{Cov}\left[\varphi_{x} \varphi_{y}\right]=g(x, y)$ to write

$$
\begin{array}{r}
\langle X, Y\rangle=\operatorname{Cov}(X, Y)=\sum_{x \in K_{1}, y \in K_{2}} \alpha_{x} \beta_{y} g(x, y), \\
\|X\|^{2}=\operatorname{Var}(X)=\sum_{x \in K_{1}, y \in K_{1}} \alpha_{x} \alpha_{y} g(x, y) .
\end{array}
$$

Also note that $P_{\mathbf{H}}\left(\varphi_{x}\right) \stackrel{1.5}{=} h_{x}, x \in \mathbb{Z}^{d}$ and that an analogous formula holds for $P_{\mathbf{K}}\left(\varphi_{x}\right)$. In particular, the entries of the matrices of $P_{\mathbf{K H}}, P_{\mathbf{H K}}$ and $A=P_{\mathbf{K H}} P_{\mathbf{H K}}$ (expressed in the basis $\varphi_{x}, x \in \mathbb{Z}^{d}$ ) are all non-negative, thus we can use the Perron-Frobenius theorem to infer that the self-adjoint matrix $A$ has an eigenvector $X_{*} \in \mathbf{H},\left\|X_{*}\right\|=1$ such that $\left\langle X_{*}, A X_{*}\right\rangle=\|A\|$ and $X_{*}$ has non-negative coordinates in the basis $\varphi_{x}, x \in K_{1}$. We claim that if we define $Y_{*}=P_{\mathbf{H K}} X_{*}$, then the pair $\left(X_{*}, Y_{*}\right)$ maximizes the correlation functional on the right-hand side of (3.4):

$$
\left\|Y_{*}\right\|=\sqrt{\left\langle P_{\mathbf{H K}} X_{*}, P_{\mathbf{H K}} X_{*}\right\rangle}=\sqrt{\left\langle X_{*}, P_{\mathbf{K H}} P_{\mathbf{H K}} X_{*}\right\rangle}=\sqrt{\|A\|} \stackrel{\sqrt[3.30]{=}}{=}\left\|P_{\mathbf{H K}}\right\|,
$$




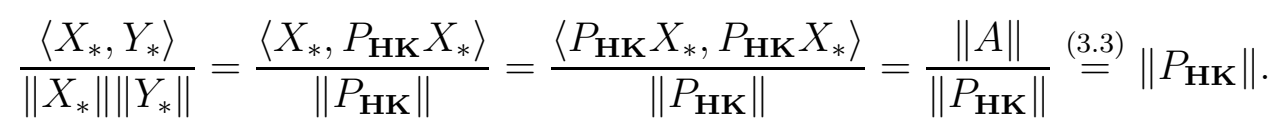

We can thus infer that the maximum in (3.4) remains unchanged if we assume

$$
\alpha_{x} \geq 0, x \in K_{1}, \quad \beta_{y} \geq 0, y \in K_{2}, \quad \sum_{x \in K_{1}} \alpha_{x}=1 \quad \text { and } \quad \sum_{y \in K_{2}} \beta_{y}=1 .
$$

Using these assumptions we can bound

$$
\begin{aligned}
c_{d}^{\prime} \operatorname{dist}\left(K_{1}, K_{2}\right)^{2-d} \stackrel{\stackrel{1.11,(1.2)}{\leq}}{\leq} \min _{x \in K_{1}, y \in K_{2}} g(x, y) \stackrel{\sqrt[(3.5),(3.7)]{\leq}}{\leq}\langle X, Y\rangle \\
\stackrel{(3.5),(3.7)}{\leq} \max _{x \in K_{1}, y \in K_{2}} g(x, y) \stackrel{\text { (1.1) }}{\leq} C_{d}^{\prime} \operatorname{dist}\left(K_{1}, K_{2}\right)^{2-d} .
\end{aligned}
$$

A combination of (3.6) and the variational characterization [9, Lemma 2.3] of capacity gives

$$
\sup _{\alpha} \frac{1}{\|X\|^{2}}=\operatorname{cap}\left(K_{1}\right), \quad \sup _{\beta} \frac{1}{\|Y\|^{2}}=\operatorname{cap}\left(K_{2}\right),
$$

where the maximum is taken over all $\alpha \in \mathbb{R}^{K_{1}}$ and $\beta \in \mathbb{R}^{K_{1}}$ satisfying (3.7). Putting together (3.4), (3.8) and (3.9) we arrive at (3.2). The proof of Proposition 1.1 is complete.

Now we prove the conditional decoupling result:

Proof of Theorem 1.2. Recall the decomposition $\varphi=\tilde{\varphi}+h$ from (1.4). Let us assume without loss of generality that our probability space is rich enough to carry an independent copy $\widehat{h}$ of the field $h$. Denote

$$
\widehat{\varphi}=\tilde{\varphi}+\widehat{h} .
$$

Clearly, $\varphi$ and $\widehat{\varphi}$ have the same law. Let $\widehat{G}_{\delta}$ be the event defined as in (1.6), but with $\widehat{h}$ replacing $h$. Then, write

$$
\begin{aligned}
\mathbb{E}\left(f_{2}(\varphi) \mid \varphi_{K_{1}}\right) \mathbb{1}_{G_{\delta}}= & \mathbb{E}\left(f_{2}(\tilde{\varphi}+h) \mid \varphi_{K_{1}}\right) \mathbb{1}_{G_{\delta}} \\
= & \mathbb{E}\left(f_{2}(\widehat{\varphi}+h-\widehat{h}) \mid \varphi_{K_{1}}\right) \mathbb{1}_{G_{\delta}} \\
= & \mathbb{E}\left(f_{2}(\widehat{\varphi}+h-\widehat{h}) \mathbb{1}_{G_{\delta} \cap \widehat{G}_{\delta}} \mid \varphi_{K_{1}}\right) \\
& \quad+\mathbb{E}\left(f_{2}(\widehat{\varphi}+h-\widehat{h}) \mathbb{1}_{G_{\delta} \cap \widehat{G}_{\delta}^{c}} \mid \varphi_{K_{1}}\right) \\
= & : T_{1}+T_{2} .
\end{aligned}
$$


Clearly, we have

$$
0 \leq T_{2} \leq \mathbb{P}\left[\widehat{G}_{\delta}^{c}\right] \mathbb{1}_{G_{\delta}}
$$

Since, by construction, $\widehat{\varphi}$ is independent of $\varphi_{K_{1}}$, moreover $|h-\widehat{h}| \leq \delta$ on $G_{\delta} \cap \widehat{G}_{\delta}$ and $f_{2}$ is increasing, we can write

$$
\begin{aligned}
T_{1} & \leq \mathbb{E}\left(f_{2}(\widehat{\varphi}+\delta) \mathbb{1}_{G_{\delta} \cap \widehat{G}_{\delta}} \mid \varphi_{K_{1}}\right) \\
& \leq \mathbb{E}\left(f_{2}(\widehat{\varphi}+\delta) \mid \varphi_{K_{1}}\right) \mathbb{1}_{G_{\delta}} \\
& =\mathbb{E}\left(f_{2}(\widehat{\varphi}+\delta)\right) \mathbb{1}_{G_{\delta}} .
\end{aligned}
$$

Also, we have

$$
\begin{aligned}
T_{1} & \geq \mathbb{E}\left(f_{2}(\widehat{\varphi}-\delta) \mathbb{1}_{\widehat{G}_{\delta}} \mid \varphi_{K_{1}}\right) \mathbb{1}_{G_{\delta}} \\
& =\mathbb{E}\left(f_{2}(\widehat{\varphi}-\delta)\left(1-\mathbb{1}_{\widehat{G}_{\delta}^{c}}\right)\right) \mathbb{1}_{G_{\delta}} \\
& \geq \mathbb{E}\left(f_{2}(\widehat{\varphi}-\delta)\right) \mathbb{1}_{G_{\delta}}-\mathbb{P}\left[\widehat{G}_{\delta}^{c}\right] \mathbb{1}_{G_{\delta}} .
\end{aligned}
$$

Inserting (3.11) - (3.13) into (3.10) and using the fact that $\widehat{\varphi}$ and $\varphi$ are equally distributed and $\mathbb{P}\left[\widehat{G}_{\delta}^{c}\right]=\mathbb{P}\left[G_{\delta}^{c}\right]$, we conclude the proof of Theorem 1.2 .

Proof of Corollary 1.3. Now, let $f_{1}: \mathbb{R}^{\mathbb{Z}^{d}} \rightarrow[0,1]$ be a function supported on $K_{1}$. Since

$$
\mathbb{E} f_{1}(\varphi)-\mathbb{P}\left[G_{\delta}^{c}\right] \leq \mathbb{E}\left(f_{1}(\varphi) \mathbb{1}_{G_{\delta}}\right) \leq \mathbb{E} f_{1}(\varphi),
$$

it is then straightforward to obtain (1.8) by multiplying (1.7) by $f_{1}(\varphi)$ and integrating.

Proof of Proposition 1.4. Define the events

$$
\Lambda_{\delta, x}=\left\{\left|h_{x}\right| \leq \delta / 2\right\}, \quad x \in H_{1}^{(=s)} .
$$

Clearly, $h_{x}$ is a centered Gaussian random variable, thus we can use (1.5) and $\mathbb{E}\left[\varphi_{x} \varphi_{y}\right]=g(x, y)$ to bound the variance of $h_{x}, x \in H_{1}^{(=s)}$ :

$$
\begin{aligned}
\operatorname{Var} h_{x} & =\sum_{y \in K_{1}} P_{x}\left[H_{K_{1}}<\infty, X_{H_{K_{1}}}=y\right] \sum_{z \in K_{1}} P_{x}\left[H_{K_{1}}<\infty, X_{H_{K_{1}}}=z\right] g(z, y) \\
& \stackrel{(*)}{=} \sum_{y \in K_{1}} P_{x}\left[H_{K_{1}}<\infty, X_{H_{K_{1}}}=y\right] g(x, y) \leq \sup _{y \in K_{1}} g(x, y) \stackrel{(1.9)}{\leq} g_{s},
\end{aligned}
$$

where $(*)$ holds by the strong Markov property of simple random walk: 


$$
\begin{aligned}
& g(x, y)=E_{x}\left[\sum_{n=0}^{\infty} \mathbb{1}_{\left[X_{n}=y\right]}\right]=E_{x}\left[\sum_{n=H_{K_{1}}}^{\infty} \mathbb{1}_{\left[X_{n}=y\right]}\right]= \\
& E_{x}\left(g\left(X_{H_{K_{1}}}, y\right) ; H_{K_{1}}<\infty\right)=\sum_{z \in K_{1}} P_{x}\left[H_{K_{1}}<\infty, X_{H_{K_{1}}}=z\right] g(z, y) .
\end{aligned}
$$

Thus we can use the exponential Chebyshev's inequality to bound

$$
\mathbb{P}\left[\Lambda_{\delta, x}^{c}\right] \leq 2 \exp \left(-\frac{\delta^{2}}{8 g_{s}}\right) .
$$

Observe that by (1.9) any nearest-neighbor walk from $K_{2}$ to $K_{1}$ must pass through $H_{1}^{(=s)}$, so by the strong Markov property of the simple random walk on $\mathbb{Z}^{d}$ and (1.5), for any $y \in K_{2}$, the value of $h_{y}$ is a weighted sum of the values $\left(h_{x}, x \in H_{1}^{(=s)}\right)$, with total weight at most 1 . In particular, we have

$$
G_{\delta}^{c} \subseteq \bigcup_{x \in H_{1}^{(=s)}} \Lambda_{\delta, x}^{c} .
$$

Using the union bound we conclude the proof of Proposition 1.4.

\section{Acknowledgements}

The work of Serguei Popov was partially supported by CNPq (300328/20052) and FAPESP (2009/52379-8). The work of Balázs Ráth is partially supported by OTKA (Hungarian National Research Fund) grant K100473, the Postdoctoral Fellowship of the Hungarian Academy of Sciences and the Bolyai Research Scholarship of the Hungarian Academy of Sciences.

The authors also thank the organizers of the conference Random Walks: Crossroads and Perspectives (Budapest, June 24-28, 2013), for providing the opportunity for the authors to meet and work on this topic. This paper was written while B.R. was a postdoctoral fellow of the University of British Columbia.

We thank Prof. Alain-Sol Sznitman for pointing out the reference [5] to us, moreover Pierre-François Rodriguez, Alexander Drewitz and a very thorough anonymous referee for reading and commenting on the manuscript.

\section{References}

[1] J. Bricmont, J.L. Lebowitz and C. Maes (1987) Percolation in strongly correlated systems: the massless Gaussian field. J. Stat. Phys. 48 (5/6), $1249-1268$. 
[2] A. Drewitz, B. Ráth, A. Sapozhnikov (2014) On chemical distances and shape theorems in percolation models with long-range correlations. $J$. Math. Phys. 55, 8, 083307.

[3] A. Drewitz, P.-F. Rodriguez (2013) High-dimensional asymptotics for percolation of Gaussian free field level sets. arXiv:1310.1041

[4] G. Grimmett (1999) Percolation, volume 321 of Grundlehren der Mathematischen Wissenschaften [Fundamental Principles of Mathematical Sciences]. Springer-Verlag, Berlin, second edition.

[5] S. Janson (1997) Gaussian Hilbert spaces. Vol. 129. Cambridge University press.

[6] G.F. Lawler (1991) Intersections of random walks. Probability and its Applications, Birkhäuser Boston Inc.

[7] T. Lupu (2014) From loop clusters and random interlacement to the free field. arXiv:1402.0298

[8] S. Popov and A. Teixeira (2013) Soft local times and decoupling of random interlacements. (to appear in J. Eur. Math. Soc), arXiv:1212.1605

[9] N.C. Jain and S. Orey (1973) Some properties of random walk paths. J. Math. Anal. Appl. 43, 795-815.

[10] P.-F. Rodriguez and A.-S. Sznitman (2013) Phase transition and levelset percolation for the Gaussian free field. Commun. Math. Phys. 320 (2), 571-601.

[11] P.-F. Rodriguez (2014) Level set percolation for random interlacements and the Gaussian free field. Stoch. Proc. Appl. 124 (4), 1469-1502.

[12] A.-S. Sznitman (2010) Vacant set of random interlacements and percolation. Ann. Math. 171, 2039-2087.

[13] A.-S. Sznitman (2012) Decoupling inequalities and interlacement percolation on $G \times \mathbb{Z}$. Invent. Math. 187 (3), 645-706.

[14] A.-S. Sznitman (2012) Topics in occupation times and Gaussian free fields. Lectures in Advanced Mathematics, EMS, Zurich.

[15] A.-S. Sznitman (2012) An isomorphism theorem for random interlacements. Electron. Commun. Probab. 17 (9), 1-9. 